\title{
Supplemental Information for: Tuning electronic structure in layered hybrid perovskites with organic spacer substitution
}

Joshua Leveillee, ${ }^{*, \dagger}, \ddagger$ Claudine Katan, $₫$ Jacky Even, ${ }^{\S}$ Dibyajyoti Ghosh, ${ }^{\ddagger}$ Wanyi Nie, ${ }^{\ddagger}$ Aditya D. Mohite, $\|$ Sergei Tretiak, ${ }^{\ddagger}$ André Schleife, ${ }^{\dagger, \perp, \#}$ and Amanda J. Neukirch*,‡

$\dagger$ Department of Materials Science and Engineering, University of Illinois at Urbana-Champaign, Urbana, IL 61801, USA $\ddagger$ Los Alamos National Laboratory, Los Alamos, NM 87545, USA

ฯUniv Rennes, ENSCR, INSA Rennes, CNRS, ISCR - UMR 6226, F-35000 Rennes, France

§Univ Rennes, INSA Rennes, CNRS, Institut FOTON - UMR 6082, F-35000 Rennes, France

||Department of Chemical and Biomolecular Engineering, Rice University, Houston, TX 77005, US

$\perp$ Materials Research Laboratory, University of Illinois at Urbana-Champaign, Urbana, IL 61801, USA \#National Center for Supercomputing Applications, University of Illinois at Urbana-Champaign, Urbana, IL 61801, USA

E-mail: leveill2@illinois.edu; ajneukirch@lanl.gov 


\section{Supporting Information Available}

\section{Notes on comparison to experiments}

In Table S1, the experimental comparisons for triplet energies for PEA and PerEA $\left(T_{1}^{*}\right)$ and fuEA and thEA $\left(T_{1}\right)$ are made to studies that examine just the optical emission from the aromatic groups without the EA tails. This is primarily due to the lack of emission data for the exact compounds available for comparison. Nonetheless, the comparisons for $T_{1}$ and $T_{1}^{*}$ are in very good agreement. Our spin charge density and natural bonding orbital analysis shows that the excitations are localized to the aromatic rings, further justifying these comparisons.

\section{Tabulated calculated data}

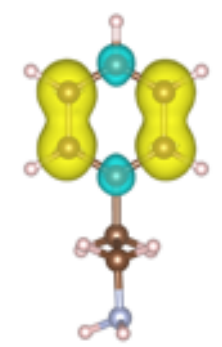

(a)

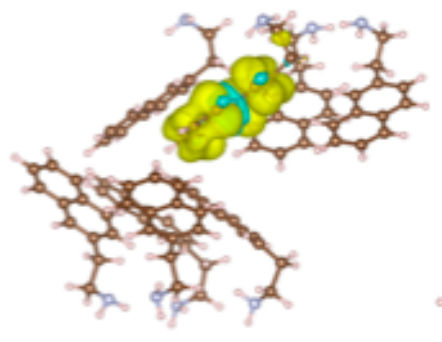

(c)

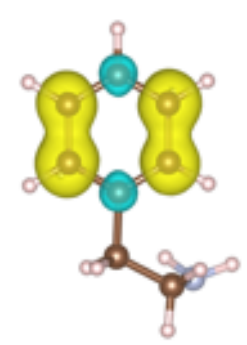

(b)

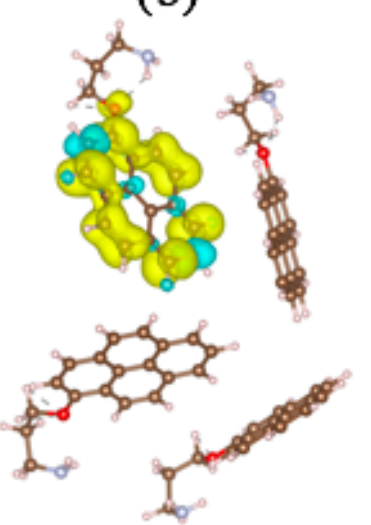

(d)

Figure S1: The magnetization density of (a) fully vacuum relaxed PEA with CAM-B3LYP, (b) relaxed phenyl ring by CAM-B3LYP with fixed EA tail in the perovskite geometry, (c) relaxed naphthalene rings on fixed EA tails in the perovskite geometry, and (d) relaxed pyrene rings on OPA tailed with fixed perovskite geometries. 
Table S1: Triplet excitation $\left(T_{1}\right)$ and emission $\left(T_{1}^{*}\right)$ energies calculated with the $\Delta \mathrm{SCF}$ method and the B3LYP XC functional. Comparison to experiment is given in the right two columns. The ${ }^{\dagger}$ symbol indicates comparisons to experiments that study only the aromatic group without the EA tail.

\begin{tabular}{|c|c|c|c|c|}
\hline Spacer & $T_{1}$ & $T_{1}^{*}$ & exp. $T_{1}$ & exp. $T_{1}^{*}$ \\
\hline PEA & 4.43 & 3.77 & & $3.40-3.60^{\dagger 11}$ \\
\hline NEA & 3.14 & 2.68 & & $2.30-2.50, \frac{{ }^{2}}{2} 2.50-2.60^{3}$ \\
\hline AEA & 2.17 & 1.75 & & $1.50-1.85^{\dagger[3} 1.80^{4}$ \\
\hline PyEA & 2.43 & 2.10 & & $1.80-2.00^{5}$ \\
\hline PerEA & 1.80 & 1.50 & & $1.56^{\dagger[6}$ \\
\hline AzEA & 2.077 & 1.75 & & \\
\hline 4Br-PEA & 4.58 & 3.20 & & \\
\hline 4Cl-PEA & 4.59 & 3.36 & & \\
\hline 4F-PEA & 4.49 & 3.57 & & \\
\hline 4MeO-PEA & 4.05 & 4.06 & & \\
\hline $4 \mathrm{NO}_{2} \mathrm{PEA}$ & 2.29 & 0.683 & & \\
\hline fuEA & 4.097 & 3.30 & $3.99^{\dagger 7}$ & \\
\hline thEA & 3.69 & 3.02 & $3.75^{\dagger 7}$ & \\
\hline selEA & 3.57 & 2.93 & & \\
\hline 2-fuEA & 2.91 & 2.40 & & \\
\hline 2-thEA & 2.68 & 2.10 & & \\
\hline 2-selEA & 2.525 & 1.970 & & \\
\hline IBfuEA & 2.36 & 1.95 & & \\
\hline IBthEA & 2.28 & 1.87 & & \\
\hline IBselEA & 2.17 & 1.77 & & \\
\hline INfuEA & 2.68 & 2.32 & & \\
\hline INthEA & 2.59 & 2.25 & & \\
\hline INselEA & 2.60 & 2.26 & & \\
\hline Azo & 1.946 & 1.215 & & \\
\hline
\end{tabular}

Table S2: Average singlet and triplet excitation energies $\left(\bar{S}_{1}\right.$ and $\bar{T}_{1}$, in eV), standard deviations $(\sigma$, in $\mathrm{eV}$ ) and bond length ranges (in $\AA$ ) on post-equilibration molecular conformations from molecular dynamics simulations of $\mathrm{A}=\mathrm{PEA}$ and $\mathrm{A}=\mathrm{NEA}$ in $\mathrm{A}_{2} \mathrm{PbI}_{4}$.

\begin{tabular}{c|ccccccc} 
& $\bar{S}_{1}$ & $\sigma_{S_{1}}$ & $S_{1, v a c}$ & $\bar{T}_{1}$ & $\sigma_{T_{1}}$ & $T_{1, v a c}$ & $\mathrm{C}-\mathrm{C}(\AA)$ \\
\hline PEA & 4.98 & 0.15 & 5.43 & 4.06 & 0.20 & 4.43 & $1.36-1.45$ \\
\hline NEA & 4.10 & 0.12 & 4.40 & 2.96 & 0.11 & 3.14 & $1.36-1.48$
\end{tabular}


Table S3: Singlet and triplet first excitation energies and homo-lumo gaps for polycarbocyclic compounds calculated by time-dependent DFT (TD) and $\triangle$ SCF methods in the singlet and triplet spin multiplicity geometries (MG) for various exchange-correlation (xc) functional. Molecular geometries herein are relaxed using the B3LYP xc-functional vacuum. $6-31 \mathrm{G}^{*}$ is used for the Gaussian basis set unless otherwise stated. All values are in $\mathrm{eV}$.

\begin{tabular}{|c|c|c|c|c|c|c|c|}
\hline & $\mathrm{MG}$ & xc-functional & $\mathrm{TD} S_{1}$ & $\mathrm{TD} T_{1}$ & $\Delta \mathrm{SCF} T_{1}$ & Homo-Lumo & Exp. $T_{1} *$ \\
\hline \multirow[t]{6}{*}{ PEA } & singlet & HSE & 5.498 & 3.54 & 4.338 & 6.054 & \\
\hline & & B3LYP & 5.429 & 3.755 & 4.433 & 6.418 & \\
\hline & & CAM-B3LYP & 5.543 & 3.508 & 4.406 & 9.292 & \\
\hline & triplet & HSE & & 2.677 & 3.714 & 3.575 & $3.4-3.6$ \\
\hline & & B3LYP & & 2.88 & 3.771 & 3.629 & \\
\hline & & CAM-B3LYP & & 2.599 & 3.763 & 3.887 & \\
\hline \multirow[t]{6}{*}{ NEA } & singlet & HSE & 4.457 & 2.563 & 3.106 & 4.391 & \\
\hline & & B3LYP & 4.396 & 2.728 & 3.136 & 4.788 & \\
\hline & & CAM-B3LYP & 4.630 & 2.524 & 3.277 & 7.315 & \\
\hline & triplet & HSE & & 1.679 & 2.654 & 3.09 & $2.3-2.5$ \\
\hline & & B3LYP & & 1.871 & 2.677 & 3.027 & \\
\hline & & CAM-B3LYP & & 1.524 & 2.761 & 3.346 & \\
\hline \multirow[t]{6}{*}{ AEA } & singlet & $\overline{\mathrm{HSE}}$ & ב3.333 & 1.685 & 2.136 & 3.202 & \\
\hline & & B3LYP & 3.293 & 1.841 & 2.172 & 3.619 & \\
\hline & & CAM-B3LYP & 3.65 & 1.603 & 2.282 & 5.953 & \\
\hline & triplet & HSE & & 0.857 & 1.724 & 2.185 & 1.8 \\
\hline & & B3LYP & & 1.093 & 1.752 & 2.142 & \\
\hline & & CAM-B3LYP & & 0.43 & 1.824 & 2.422 & \\
\hline \multirow[t]{6}{*}{ PyEA } & singlet & $\overline{\mathrm{HSE}}$ & 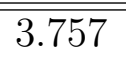 & 1.977 & 2.402 & 3.435 & \\
\hline & & B3LYP & 3.705 & 2.121 & 2.434 & 3.84 & \\
\hline & & CAM-B3LYP & 3.964 & 1.866 & 2.552 & 6.089 & \\
\hline & triplet & HSE & & 1.302 & 2.076 & 2.560 & $1.8-2.0$ \\
\hline & & B3LYP & & 1.474 & 2.101 & 2.509 & \\
\hline & & CAM-B3LYP & & 0.951 & 2.161 & 2.898 & \\
\hline \multirow[t]{6}{*}{$\overline{\text { PerEA }}$} & singlet & HSE & 2.892 & 1.393 & 1.770 & 2.611 & \\
\hline & & B3LYP & 2.855 & 1.526 & 1.802 & 3.028 & \\
\hline & & CAM-B3LYP & 3.177 & 1.306 & 1.955 & 5.194 & \\
\hline & triplet & HSE & & 0.722 & 1.47 & 1.808 & \\
\hline & & B3LYP & & 0.913 & 1.504 & 1.78 & \\
\hline & & CAM-B3LYP & & -0.185 & 1.598 & 2.108 & \\
\hline
\end{tabular}


Table S4: Singlet and triplet first excitation energies and homo-lumo gaps for functionalized 4Z-PEA compounds calculated by time-dependent DFT (TD) and $\triangle$ SCF methods in the singlet and triplet spin multiplicity geometries (MG) for various exchange-correlation (xc) functional. Molecular geometries herein are relaxed using the B3LYP xc-functional vacuum. $6-31 \mathrm{G}^{*}$ is used for the Gaussian basis set unless otherwise stated. All values are in eV.

\begin{tabular}{|c|c|c|c|c|c|c|}
\hline Compound & MG & xc-functional & $\mathrm{TD} S_{1}$ & $\mathrm{TD} T_{1}$ & $\triangle \mathrm{SCF} T_{1}$ & Homo-Lumo \\
\hline \multirow[t]{6}{*}{ 4Br-PEA } & singlet & HSE & 5.156 & 3.448 & 4.570 & 5.482 \\
\hline & & B3LYP & 5.083 & 3.64 & 4.578 & 5.853 \\
\hline & & CAM-B3LYP & 5.363 & 3.4249 & 4.7489 & 8.643 \\
\hline & triplet & $\mathrm{HSE}$ & & -0.436 & 3.262 & \\
\hline & & B3LYP & & 0.391 & 3.202 & \\
\hline & & CAM-B3LYP & & -0.554 & 3.386 & \\
\hline \multirow[t]{6}{*}{ 4Cl-PEA } & singlet & $\overline{\mathrm{HSE}}$ & $\overline{5.197}$ & 3.455 & 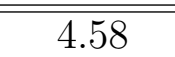 & 5.517 \\
\hline & & B3LYP & 5.123 & 3.648 & 4.59 & 5.886 \\
\hline & & CAM-B3LYP & 5.381 & 3.423 & 4.745 & 8.727 \\
\hline & triplet & HSE & & 0.462 & 3.353 & \\
\hline & & B3LYP & & 0.833 & 3.359 & \\
\hline & & CAM-B3LYP & & -0.26 & 3.44 & \\
\hline \multirow[t]{6}{*}{ 4F-PEA } & singlet & HSE & 5.253 & 3.556 & 4.4749 & 5.514 \\
\hline & & B3LYP & 5.18 & 3.753 & 4.493 & 5.884 \\
\hline & & CAM-B3LYP & 5.423 & 3.515 & 4.605 & 8.717 \\
\hline & triplet & HSE & & -0.570 & 3.535 & \\
\hline & & B3LYP & & 0.471 & 3.573 & \\
\hline & & CAM-B3LYP & & -0.851 & 3.577 & \\
\hline \multirow[t]{6}{*}{ 4MeO-PEA } & singlet & HSE & 5.149 & 3.519 & 3.987 & 5.429 \\
\hline & & B3LYP & 5.08 & 3.697 & 4.05 & 5.810 \\
\hline & & CAM-B3LYP & 5.248 & 3.476 & 4.368 & 8.523 \\
\hline & triplet & HSE & & 3.245 & 4.051 & \\
\hline & & B3LYP & & 3.44 & 4.054 & \\
\hline & & CAM-B3LYP & & 3.173 & 4.163 & \\
\hline \multirow[t]{6}{*}{ 4NO2-PEA } & singlet & $\overline{\mathrm{HSE}}$ & 3.031 & 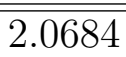 & 2.254 & 3.398 \\
\hline & & B3LYP & 2.9653 & 2.136 & 2.287 & 3.695 \\
\hline & & CAM-B3LYP & 3.192 & 2.091 & 2.254 & 6.709 \\
\hline & triplet & HSE & & -0.576 & 0.788 & \\
\hline & & B3LYP & & -0.523 & 0.683 & \\
\hline & & CAM-B3LYP & & -0.807 & 0.683 & \\
\hline
\end{tabular}


Table S5: Singlet and triplet first excitation energies and homo-lumo gaps for single and linked heterocyclic ring compounds calculated by time-dependent DFT (TD) and $\triangle \mathrm{SCF}$ methods in the singlet and triplet spin multiplicity geometries (MG) for various exchangecorrelation (xc) functional. Molecular geometries herein are relaxed using the B3LYP xcfunctional vacuum. 6-31G* is used for the Gaussian basis set unless otherwise stated. All values are in $\mathrm{eV}$.

\begin{tabular}{|c|c|c|c|c|c|c|}
\hline Compound & MG & xc-functional & $\mathrm{TD} S_{1}$ & $\mathrm{TD} T_{1}$ & $\Delta \mathrm{SCF} T_{1}$ & Homo-Lumo \\
\hline \multirow[t]{6}{*}{ fuEA } & singlet & HSE & 6.255 & 3.616 & 4.034 & 6.052 \\
\hline & & B3LYP & 6.177 & 3.749 & 4.097 & 6.403 \\
\hline & & CAM-B3LYP & 6.326 & 3.617 & 4.107 & 9.305 \\
\hline & triplet & HSE & & 1.279 & 3.265 & \\
\hline & & B3LYP & & 1.531 & 3.305 & \\
\hline & & CAM-B3LYP & & 1.068 & 3.318 & \\
\hline \multirow[t]{6}{*}{ 2-fuEA } & singlet & HSE & 4.433 & 2.497 & 2.882 & 4.147 \\
\hline & & B3LYP & 4.380 & 2.597 & 2.908 & 4.534 \\
\hline & & CAM-B3LYP & 4.6254 & 2.498 & 3.038 & 7.138 \\
\hline & triplet & HSE & & 1.463 & 2.405 & \\
\hline & & B3LYP & & 1.2497 & 2.401 & \\
\hline & & CAM-B3LYP & & & 2.495 & \\
\hline \multirow[t]{6}{*}{ thEA } & singlet & HSE & 5.424 & 3.236 & 3.634 & 5.370 \\
\hline & & B3LYP & 5.336 & 3.353 & 3.686 & 5.734 \\
\hline & & CAM-B3LYP & 5.708 & 3.210 & 3.690 & 8.547 \\
\hline & triplet & HSE & & 1.204 & 3.005 & \\
\hline & & B3LYP & & 1.4232 & 3.0266 & \\
\hline & & CAM-B3LYP & & 0.938 & 3.0326 & \\
\hline \multirow[t]{6}{*}{ 2-thEA } & singlet & $\overline{\mathrm{HSE}}$ & 3.996 & 2.269 & 2.667 & $\overline{3.814}$ \\
\hline & & B3LYP & 3.9417 & 2.361 & 2.683 & 4.204 \\
\hline & & CAM-B3LYP & 4.211 & 2.242 & 2.817 & 6.721 \\
\hline & triplet & HSE & & 1.150 & 2.111 & \\
\hline & & B3LYP & & 1.290 & 2.096 & \\
\hline & & CAM-B3LYP & & 0.809 & 2.180 & \\
\hline \multirow[t]{6}{*}{ selEA } & singlet & HSE & 5.269 & 3.114 & 3.520 & 5.243 \\
\hline & & B3LYP & 5.185 & 3.236 & 3.574 & 5.613 \\
\hline & & CAM-B3LYP & 5.507 & 3.086 & 3.578 & 8.402 \\
\hline & triplet & HSE & & 1.037 & 2.906 & \\
\hline & & B3LYP & & 1.285 & 2.932 & \\
\hline & & CAM-B3LYP & & 0.713 & 2.934 & \\
\hline \multirow[t]{6}{*}{2 -selEA } & singlet & HSE & 3.805 & 2.106 & 2.504 & 3.635 \\
\hline & & B3LYP & 3.7563 & 2.2039 & 2.525 & 4.029 \\
\hline & & CAM-B3LYP & 4.010 & 2.061 & 2.641 & 6.510 \\
\hline & triplet & HSE & & 1.000 & 1.982 & \\
\hline & & B3LYP & & 1.154 & 1.970 & \\
\hline & & CAM-B3LYP & & 0.530 & 2.043 & \\
\hline
\end{tabular}


Table S6: Singlet and triplet first excitation energies and homo-lumo gaps for fused heterocyclic ring compounds calculated by time-dependent DFT (TD) and $\triangle \mathrm{SCF}$ methods in the singlet and triplet spin multiplicity geometries (MG) for various exchange-correlation (xc) functional. Molecular geometries herein are relaxed using the B3LYP xc-functional vacuum. $6-31 \mathrm{G}^{*}$ is used for the Gaussian basis set unless otherwise stated. All values are in $\mathrm{eV}$.

\begin{tabular}{|c|c|c|c|c|c|c|}
\hline Compound & MG & xc-functional & $\mathrm{TD} S_{1}$ & $\mathrm{TD} T_{1}$ & $\Delta \mathrm{SCF} T_{1}$ & Homo-Lumo \\
\hline \multirow[t]{6}{*}{ IBfuEA } & singlet & HSE & 3.847 & 1.878 & 2.302 & 3.702 \\
\hline & & B3LYP & 3.802 & 2.034 & 2.360 & 4.113 \\
\hline & & CAM-B3LYP & 4.078 & 1.813 & 2.412 & 6.656 \\
\hline & triplet & HSE & & 0.903 & 1.912 & \\
\hline & & B3LYP & & 1.156 & 1.949 & \\
\hline & & CAM-B3LYP & & 0.514 & 1.982 & \\
\hline \multirow[t]{6}{*}{ INfuEA } & singlet & HSE & 3.936 & 2.227 & 2.650 & 3.795 \\
\hline & & B3LYP & 3.881 & 2.361 & 2.680 & 4.197 \\
\hline & & CAM-B3LYP & 4.243 & 2.183 & 2.810 & 6.621 \\
\hline & triplet & HSE & & 1.507 & 2.301 & \\
\hline & & B3LYP & & 1.672 & 2.330 & \\
\hline & & CAM-B3LYP & & 1.330 & 2.416 & \\
\hline \multirow[t]{6}{*}{ IBthEA } & singlet & HSE & 3.750 & 1.816 & 2.234 & 3.571 \\
\hline & & B3LYP & 3.696 & 1.951 & 2.276 & 3.977 \\
\hline & & CAM-B3LYP & 3.958 & 1.714 & 2.304 & 6.450 \\
\hline & triplet & HSE & & 0.818 & 1.856 & \\
\hline & & B3LYP & & 1.053 & 1.869 & \\
\hline & & CAM-B3LYP & & 0.206 & 1.885 & \\
\hline \multirow[t]{6}{*}{ INthEA } & ב singlet & $\overline{\mathrm{HSE}}$ & $\overline{3.712}$ & 2.131 & 2.554 & (3.623 \\
\hline & & B3LYP & 3.667 & 2.268 & 2.587 & 4.033 \\
\hline & & CAM-B3LYP & 4.065 & 2.095 & 2.736 & 6.434 \\
\hline & triplet & HSE & & 1.421 & 2.206 & \\
\hline & & B3LYP & & 1.591 & 2.246 & \\
\hline & & CAM-B3LYP & & 1.246 & 2.344 & \\
\hline \multirow[t]{6}{*}{ IBselEA } & singlet & HSE & 3.604 & 1.700 & 2.122 & 3.442 \\
\hline & & B3LYP & 3.552 & 1.835 & 2.166 & 3.849 \\
\hline & & CAM-B3LYP & 3.803 & 1.583 & 2.182 & 3.301 \\
\hline & triplet & HSE & & 0.670 & 1.753 & \\
\hline & & B3LYP & & 0.932 & 1.767 & \\
\hline & & CAM-B3LYP & & -0.438 & 1.776 & \\
\hline \multirow[t]{6}{*}{ INselEA } & singlet & HSE & 3.627 & 2.140 & 2.562 & 3.578 \\
\hline & & B3LYP & 3.587 & 2.275 & 2.595 & 3.992 \\
\hline & & CAM-B3LYP & 4.018 & 2.115 & 2.759 & 6.392 \\
\hline & triplet & $\mathrm{HSE}$ & & 1.442 & 2.216 & \\
\hline & & B3LYP & & 1.609 & 2.257 & \\
\hline & & CAM-B3LYP & & 1.282 & 2.368 & \\
\hline
\end{tabular}


Table S7: Singlet and triplet first excitation energies and homo-lumo gaps for azobenzeneEA (Azo) calculated by time-dependent DFT (TD) and $\triangle \mathrm{SCF}$ methods in the singlet and triplet spin multiplicity geometries (MG) for various exchange-correlation (xc) functional. Molecular geometries herein are relaxed using the B3LYP xc-functional vacuum. 6-31G* is used for the Gaussian basis set unless otherwise stated. All values are in $\mathrm{eV}$.

\begin{tabular}{c|c|c|c|c|c|c}
\hline Compound & MG & xc-functional & TD $S_{1}$ & TD $T_{1}$ & $\Delta$ SCF $T_{1}$ & Homo-Lumo \\
\hline \multirow{2}{*}{ Azo } & singlet & HSE & 2.579 & 1.733 & 1.900 & 3.523 \\
& & B3LYP & 2.562 & 1.793 & 1.946 & 3.907 \\
& & CAM-B3LYP & & 2.726 & 1.971 & 6.399 \\
\hline \multirow{3}{*}{ triplet } & HSE & & -0.2743 & 1.174 & \\
& & B3LYP & & -0.317 & 1.215 & \\
& & CAM-B3LYP & & -0.3048 & 1.233 & \\
\hline
\end{tabular}


Table S8: Singlet and triplet first excitation energies and homo-lumo gaps for polycarbocyclic compounds calculated by time-dependent DFT (TD) and $\triangle$ SCF methods in the singlet and triplet spin multiplicity geometries (MG) for various exchange-correlation (xc) functional. Molecular geometries herein are relaxed using the CAM-B3LYP xc-functional. Values outside of parentheses indicate the neutral compound with an ethyl ammonia $\mathrm{NH}_{2}$ tail whereas values in the parentheses indicate the charged species with $\mathrm{NH}_{3}^{+}$. 6-31G ${ }^{*}$ is used for the Gaussian basis set unless otherwise stated. All values are in $\mathrm{eV}$. The $\dagger$ symbol indicates instances where the xc-functional cannot appropriately handle range-separation for charged species (HSE and B3LYP).

\begin{tabular}{|c|c|c|c|c|c|c|}
\hline & $\mathrm{MG}$ & xc-functional & $\mathrm{TD} S_{1}$ & $\overline{\mathrm{TD}} T_{1}$ & $\Delta \mathrm{SCF} T_{1}$ & Homo-Lumo \\
\hline \multirow[t]{6}{*}{ PEA } & singlet & HSE & $5.547\left(5.088^{\dagger}\right)$ & $3.604(3.595)$ & $4.389(4.485)$ & $6.060(5.239)$ \\
\hline & & B3LYP & $5.478\left(4.745^{\dagger}\right)$ & $3.815(3.571)$ & $4.840(4.374)$ & $6.422(5.323)$ \\
\hline & & CAM-B3LYP & $5.594(5.581)$ & $3.576(3.571)$ & $4.480(5.199)$ & $9.340(8.090)$ \\
\hline & triplet & HSE & & $0.788(2.686)$ & $1.549(3.095)$ & \\
\hline & & B3LYP & & $1.158(2.882)$ & $1.669(3.196)$ & \\
\hline & & CAM-B3LYP & & $0.548(2.613)$ & $1.551(2.083)$ & \\
\hline \multirow[t]{6}{*}{ NEA } & singlet & HSE & $4.518\left(3.902^{\dagger}\right)$ & $2.642(2.623)$ & $3.181(3.138)$ & $4.462(3.970)$ \\
\hline & & B3LYP & $4.457\left(3.607^{\dagger}\right)$ & $2.804(2.779)$ & $3.209(3.165)$ & $4.859(4.067)$ \\
\hline & & CAM-B3LYP & $4.696(4.645)$ & $2.615(2.601)$ & $3.362(3.318)$ & $7.399(6.705)$ \\
\hline & triplet & HSE & & $1.648(1.661)$ & $2.148(2.144)$ & \\
\hline & & B3LYP & & $1.839(1.846)$ & $2.204(2.198)$ & \\
\hline & & CAM-B3LYP & & $1.487(1.508)$ & $2.196(2.195)$ & \\
\hline \multirow[t]{6}{*}{$\overline{\mathrm{AEA}}$} & singlet & $\overline{\mathrm{HSE}}$ & $3.401\left(3.085^{\dagger}\right)$ & $\bar{~} 1.772(1.709)$ & 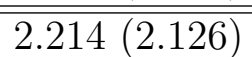 & "3.280(3.108) \\
\hline & & B3LYP & $3.360\left(2.841^{\dagger}\right)$ & $1.923(1.853)$ & $2.248(2.160)$ & $3.694(3.236)$ \\
\hline & & CAM-B3LYP & $3.728(3.563)$ & $1.712(1.654)$ & $2.372(2.286)$ & $6.045(5.734)$ \\
\hline & triplet & HSE & & $0.804(0.826)$ & $1.376(1.358)$ & \\
\hline & & B3LYP & & $1.048(1.056)$ & $1.428(1.416)$ & \\
\hline & & CAM-B3LYP & & $0.289(0.383)$ & $1.394(1.386)$ & \\
\hline \multirow[t]{6}{*}{ PyEA } & singlet & HSE & $3.829\left(3.246^{\dagger}\right)$ & $2.073(2.074)$ & $2.494(2.478)$ & $3.533(3.292)$ \\
\hline & & B3LYP & $3.776\left(2.976^{\dagger}\right)$ & $2.212(2.209)$ & $2.523(2.507)$ & $3.926(3.385)$ \\
\hline & & CAM-B3LYP & $4.040(3.982)$ & $1.986(1.989)$ & $2.661(2.641)$ & $6.195(5.878)$ \\
\hline & triplet & HSE & & $1.259(1.307)$ & $1.682(1.708)$ & \\
\hline & & B3LYP & & $1.431(1.472)$ & $1.734(1.760)$ & \\
\hline & & CAM-B3LYP & & $0.878(0.967)$ & $1.672(1.700)$ & \\
\hline \multirow[t]{6}{*}{ PerEA } & singlet & HSE & $2.967(2.855)$ & $1.497(1.4838)$ & $1.867(1.822)$ & $2.702(2.616)$ \\
\hline & & B3LYP & $2.932(2.619)$ & $1.624(1.599)$ & $1.896(1.848)$ & $3.118(3.009)$ \\
\hline & & CAM-B3LYP & 3.266 (3.177) & $1.444(1.429)$ & $2.073(2.017)$ & $5.309(5.197)$ \\
\hline & triplet & HSE & & $0.667(0.765)$ & $1.121(1.156)$ & \\
\hline & & B3LYP & & $0.863(0.931)$ & $1.170(1.201)$ & \\
\hline & & CAM-B3LYP & & $-0.361^{*}(0.218)$ & $1.140(1.179)$ & \\
\hline
\end{tabular}


Table S9: Singlet and triplet first excitation energies and homo-lumo gaps for functionalized 4Z-PEA compounds calculated by time-dependent DFT (TD) and $\triangle$ SCF methods in the singlet and triplet spin multiplicity geometries (MG) for various exchange-correlation (xc) functional. Molecular geometries herein are relaxed using the CAM-B3LYP xc-functional. Values outside of parentheses indicate the neutral compound with an ethyl ammonia $\mathrm{NH}_{2}$ tail whereas values in the parentheses indicate the charged species with $\mathrm{NH}_{3}^{+} \cdot 6-31 \mathrm{G}^{*}$ is used for the Gaussian basis set unless otherwise stated. All values are in $\mathrm{eV}$.

\begin{tabular}{|c|c|c|c|c|c|c|}
\hline & $\mathrm{MG}$ & xc-functional & $\mathrm{TD} S_{1}$ & $\mathrm{TD} T_{1}$ & $\Delta \mathrm{SCF} T_{1}$ & Homo-Lumo \\
\hline \multirow[t]{6}{*}{ MeO-PEA } & singlet & HSE & $5.189\left(4.110^{\dagger}\right)$ & $3.582(3.556)$ & $4.038(3.951)$ & $5.466(4.222)$ \\
\hline & & B3LYP & $5.120\left(3.800^{\dagger}\right)$ & $3.757(3.694)$ & $4.100(3.993)$ & $5.847(4.319)$ \\
\hline & & CAM-B3LYP & $5.288(5.230)$ & $3.545(3.531)$ & $4.404(4.394)$ & $8.563(7.037)$ \\
\hline & triplet & $\mathrm{HSE}$ & & $3.498(3.306)$ & $3.832(3.651)$ & \\
\hline & & B3LYP & & $3.241(3.467)$ & $3.855(3.671)$ & \\
\hline & & CAM-B3LYP & & $3.306(3.246)$ & $3.924(3.796)$ & \\
\hline \multirow[t]{6}{*}{ NO2-PEA } & singlet & $\overline{\mathrm{HSE}}$ & $3.250(3.365)$ & $2.132(2.197)$ & $2.315(2.375)$ & $3.664(4.348)$ \\
\hline & & B3LYP & $3.205(3.346)$ & $2.216(2.274)$ & $2.354(2.414)$ & $3.953(4.637)$ \\
\hline & & CAM-B3LYP & $3.314(3.358)$ & $2.147(2.196)$ & $2.315(2.364)$ & $7.011(7.587)$ \\
\hline & triplet & HSE & & $1.619(1.623)$ & $1.828(1.835)$ & \\
\hline & & B3LYP & & $1.7245(1.726)$ & $1.879(1.884)$ & \\
\hline & & CAM-B3LYP & & $1.628(1.640)$ & $1.819(1.832)$ & \\
\hline \multirow[t]{12}{*}{ Br-PEA } & singlet & $\overline{\mathrm{HSE}}$ & $5.185(4.521)$ & $3.507(3.461)$ & $4.601(4.388)$ & $5.499(4.607)$ \\
\hline & & B3LYP & $5.111(4.222)$ & $3.694(3.631)$ & $4.609(4.081)$ & $5.870(4.693)$ \\
\hline & & CAM-B3LYP & $5.401(5.353)$ & $3.488(3.451)$ & $4.781(4.837)$ & $8.678(7.442)$ \\
\hline & singlet & HSE & 4.837 & 3.489 & 4.575 & \\
\hline & $\operatorname{lanl} 2 \mathrm{dz}$ & B3LYP & 4.740 & 3.643 & 4.546 & \\
\hline & & CAM-B3LYP & 5.306 & 3.421 & 4.770 & \\
\hline & triplet & HSE & & $2.633(3.159)$ & $3.043(3.765)$ & \\
\hline & & B3LYP & & $2.817(3.355)$ & $3.130(4.103)$ & \\
\hline & & CAM-B3LYP & & $2.570(3.111)$ & $3.088(4.286)$ & \\
\hline & triplet & HSE & & 3.091 & 3.949 & \\
\hline & $\operatorname{lanl} 2 \mathrm{dz}$ & B3LYP & & 3.266 & 3.919 & \\
\hline & & CAM-B3LYP & & 2.989 & 4.165 & \\
\hline \multirow[t]{6}{*}{ Cl-PEA } & singlet & $\overline{\mathrm{HSE}}$ & $5.282(4.728)$ & $3.620(3.486)$ & $4.403(4.723)$ & $5.532(4.837)$ \\
\hline & & B3LYP & $5.208(4.413)$ & $3.815(3.664)$ & $4.525(4.131)$ & $5.901(4.926)$ \\
\hline & & CAM-B3LYP & $5.466(5.404)$ & $3.584(3.461)$ & $4.540(4.865)$ & $8.745(7.677)$ \\
\hline & triplet & HSE & & $0.380(0.520)$ & $1.245(1.316)$ & \\
\hline & & B3LYP & & $0.805(0.897)$ & $1.339(1.410)$ & \\
\hline & & CAM-B3LYP & & $-0.429(-0.310)$ & $1.262(1.315)$ & \\
\hline \multirow[t]{6}{*}{$\overline{\text { F-PEA }}$} & singlet & $\overline{\mathrm{HSE}}$ & $5.282(4.855)$ & $3.620(3.624)$ & $4.403(4.679)$ & $5.532(4.994)$ \\
\hline & & B3LYP & $5.208(4.520)$ & $3.815(3.810)$ & $4.525(4.689)$ & $5.901(5.082)$ \\
\hline & & CAM-B3LYP & $5.466(5.404)$ & $3.584(3.461)$ & $4.640(4.865)$ & $8.745(7.677)$ \\
\hline & triplet & HSE & & $-2.091(-2.212)$ & $0.654(0.947)$ & \\
\hline & & B3LYP & & $-1.924(-2.037)$ & $0.378(0.618)$ & \\
\hline & & CAM-B3LYP & & $-2.262(-2.392)$ & $0.813(1.121)$ & \\
\hline
\end{tabular}


Table S10: Singlet and triplet first excitation energies and homo-lumo gaps for single and linked heterocyclic ring compounds calculated by time-dependent DFT (TD) and $\triangle$ SCF methods in the singlet and triplet spin multiplicity geometries $(\mathrm{MG})$ for various exchangecorrelation (xc) functional. Molecular geometries herein are relaxed using the CAMB3LYP xc-functional. Values outside of parentheses indicate the neutral compound with an ethyl ammonia $\mathrm{NH}_{2}$ tail whereas values in the parentheses indicate the charged species with $\mathrm{NH}_{3}^{+}$. 6-31G* is used for the Gaussian basis set unless otherwise stated. All values are in $\mathrm{eV}$.

\begin{tabular}{|c|c|c|c|c|c|c|}
\hline & MG & xc-functional & $\mathrm{TD} S_{1}$ & $\mathrm{TD} T_{1}$ & $\Delta \mathrm{SCF} T_{1}$ & Homo-Lumo \\
\hline \multirow[t]{6}{*}{ selEA } & singlet & HSE & $5.339(4.637)$ & $3.222(3.231)$ & $3.620(3.621)$ & $5.328(4.785)$ \\
\hline & & B3LYP & $5.254(4.291)$ & $3.340(3.348)$ & $3.673(3.675)$ & $5.696(4.872)$ \\
\hline & & CAM-B3LYP & $5.601(5.483)$ & $3.201(3.201)$ & $3.682(3.674)$ & $8.499(7.656)$ \\
\hline & triplet & $\mathrm{HSE}$ & & $0.995(0.773)$ & $1.630(1.454)$ & \\
\hline & & B3LYP & & $1.248(1.070)$ & $1.726(1.561)$ & \\
\hline & & CAM-B3LYP & & $0.658(0.237)$ & $1.585(1.408)$ & \\
\hline \multirow[t]{6}{*}{ 2-selEA } & singlet & HSE & $3.933(3.376)$ & $2.280(2.258)$ & $2.675(2.633)$ & $3.800(3.447)$ \\
\hline & & B3LYP & $3.8805(3.101)$ & $2.372(2.340)$ & $2.692(2.646)$ & $4.191(3.536)$ \\
\hline & & CAM-B3LYP & $4.1564(4.046)$ & $2.260(2.238)$ & $2.833(2.785)$ & $5.294(6.184)$ \\
\hline & triplet & HSE & & $0.997(1.061)$ & $1.446(1.469)$ & \\
\hline & & B3LYP & & 1.148 (1.198) & $1.491(1.512)$ & \\
\hline & & CAM-B3LYP & & $0.517(0.638)$ & $1.385(1.407)$ & \\
\hline \multirow[t]{6}{*}{ thEA } & singlet & HSE & $5.480(4.658)$ & $3.336(3.345)$ & $3.728(3.725)$ & $5.447(4.817)$ \\
\hline & & B3LYP & $5.389(4.3117)$ & $3.450(3.316)$ & $3.778(3.778)$ & $5.810(4.904)$ \\
\hline & & CAM-B3LYP & $5.791(5.732)$ & $3.318(3.316)$ & $3.787(3.776)$ & 8.635 (7.709) \\
\hline & triplet & HSE & & $1.162(1.175)$ & $1.735(1.726)$ & \\
\hline & & B3LYP & & 1.385 (1.401) & $1.827(1.826)$ & \\
\hline & & CAM-B3LYP & & $0.891(0.924)$ & $1.689(1.691)$ & \\
\hline \multirow[t]{6}{*}{ 2-thEA } & singlet & $\overline{\mathrm{HSE}}$ & $4.130(3.417)$ & $\overline{2.449(2.422)}$ & $\bar{~} 2.846(2.803)$ & $3.987(3.487)$ \\
\hline & & B3LYP & $4.073(3.140)$ & $2.535(2.450)$ & $2.859(2.811)$ & $4.374(3.578)$ \\
\hline & & CAM-B3LYP & $4.368(4.253)$ & 2.443 (2.419) & $3.017(2.970)$ & $6.920(6.238)$ \\
\hline & triplet & $\mathrm{HSE}$ & & $1.139(1.196)$ & $1.563(1.586)$ & \\
\hline & & B3LYP & & $1.277(1.323)$ & $1.605(1.626)$ & \\
\hline & & CAM-B3LYP & & $0.786(0.867)$ & $1.521(1.542)$ & \\
\hline \multirow[t]{6}{*}{ fuEA } & singlet & HSE & $6.328(4.476)$ & $3.709(3.629)$ & $4.121(4.022)$ & $6.140(4.638)$ \\
\hline & & B3LYP & $6.244(4.142)$ & 3.839 (3.636) & $4.184(4.081)$ & $6.489(4.745)$ \\
\hline & & CAM-B3LYP & $6.408(5.584)$ & $3.714(3.634)$ & $4.197(4.106)$ & $9.398(7.565)$ \\
\hline & triplet & HSE & & $1.325(1.569)$ & $1.908(2.084)$ & \\
\hline & & B3LYP & & $1.570(1.796)$ & $2.023(2.200)$ & \\
\hline & & CAM-B3LYP & & $1.123(1.394)$ & $1.898(2.078)$ & \\
\hline \multirow[t]{6}{*}{ 2-fuEA } & singlet & HSE & $4.529(3.210)$ & $2.621(2.566)$ & $3.003(2.938)$ & $4.625(3.290)$ \\
\hline & & B3LYP & $4.475(2.929)$ & $2.717(2.656)$ & 3.026 (2.957) & $4.650(3.401)$ \\
\hline & & CAM-B3LYP & $4.732(4.484)$ & $2.636(2.583)$ & 3.169 (3.107) & $7.273(6.063)$ \\
\hline & triplet & HSE & & $1.455(1.447)$ & $1.853(1.832)$ & \\
\hline & & B3LYP & & $1.595(1.584)$ & $1.904(1.882)$ & \\
\hline & & CAM-B3LYP & & $1.231(1.217)$ & $1.843(1.822)$ & \\
\hline
\end{tabular}


Table S11: Singlet and triplet first excitation energies and homo-lumo gaps for fused heterocyclic ring compounds calculated by time-dependent DFT (TD) and $\triangle$ SCF methods in the singlet and triplet spin multiplicity geometries (MG) for various exchange-correlation (xc) functional. Molecular geometries herein are relaxed using the CAM-B3LYP xc-functional. Values outside of parentheses indicate the neutral compound with an ethyl ammonia $\mathrm{NH}_{2}$ tail whereas values in the parentheses indicate the charged species with $\mathrm{NH}_{3}^{+} \cdot 6-31 \mathrm{G}^{*}$ is used for the Gaussian basis set unless otherwise stated. All values are in eV.

\begin{tabular}{|c|c|c|c|c|c|c|}
\hline & $\mathrm{MG}$ & xc-functional & $\mathrm{TD} S_{1}$ & $\mathrm{TD} T_{1}$ & $\Delta \mathrm{SCF} T_{1}$ & Homo-Lumo \\
\hline \multirow[t]{6}{*}{ IBSelEA } & \multirow[t]{3}{*}{ singlet } & HSE & $3.693(3.461)$ & $1.824(1.823)$ & $2.233(2.235)$ & $3.543(3.500)$ \\
\hline & & B3LYP & $3.640(3.170)$ & $1.952(1.979)$ & $2.273(2.276)$ & $3.949(3.637)$ \\
\hline & & CAM-B3LYP & $3.904(3.822)$ & $1.733(1.776)$ & $2.305(2.306)$ & $6.417(6.262)$ \\
\hline & \multirow[t]{3}{*}{ triplet } & HSE & & $0.690(0.832)$ & $1.319(1.371)$ & \\
\hline & & B3LYP & & $0.943(1.043)$ & $1.387(1.438)$ & \\
\hline & & CAM-B3LYP & & $-0.412(0.287)$ & $1.273(1.333)$ & \\
\hline \multirow[t]{6}{*}{ INSelEA } & \multirow[t]{3}{*}{ singlet } & HSE & $3.674(3.080)$ & $2.210(2.151)$ & $2.623(2.513)$ & $3.634(3.116)$ \\
\hline & & B3LYP & $3.633(2.849)$ & $2.341(2.263)$ & $2.655(2.541)$ & $4.048(3.221)$ \\
\hline & & CAM-B3LYP & $4.072(3.788)$ & $2.202(2.165)$ & $2.827(2.726)$ & $6.460(5.782)$ \\
\hline & \multirow[t]{3}{*}{ triplet } & HSE & & $1.400(1.418)$ & $1.831(1.804)$ & \\
\hline & & B3LYP & & $1.570(1.568)$ & $1.885(1.853)$ & \\
\hline & & CAM-B3LYP & & $1.230(1.275)$ & $1.910(1.896)$ & \\
\hline \multirow[t]{6}{*}{ IBThEA } & \multirow[t]{3}{*}{ singlet } & HSE & $3.833(3.520)$ & $1.932(1.958)$ & $2.338(2.327)$ & $3.668(3.576)$ \\
\hline & & B3LYP & $3.772(3.229)$ & $2.059(2.075)$ & $2.378(2.367)$ & $4.071(3.695)$ \\
\hline & & CAM-B3LYP & $4.052(3.943)$ & $1.851(1.883)$ & $2.420(2.410)$ & $6.560(6.324)$ \\
\hline & \multirow[t]{3}{*}{ triplet } & HSE & & $0.826(0.936)$ & $1.409(1.447)$ & \\
\hline & & B3LYP & & 1.0567 (1.138) & $1.477(1.514)$ & \\
\hline & & CAM-B3LYP & & $0.223(0.534)$ & $1.376(1.423)$ & \\
\hline \multirow[t]{6}{*}{ INThEA } & \multirow[t]{3}{*}{ singlet } & $\overline{\mathrm{HSE}}$ & $3.764(3.197)$ & $20.203(2.159)$ & "2.618(2.528) & "3.683 (3.238) \\
\hline & & B3LYP & $3.718(2.952)$ & $2.336(2.276)$ & $2.650(2.556)$ & $4.093(3.340)$ \\
\hline & & CAM-B3LYP & $4.124(3.879)$ & $2.1831(2.155)$ & $2.807(2.724)$ & $6.507(5.907)$ \\
\hline & \multirow[t]{3}{*}{ triplet } & HSE & & $1.380(1.407)$ & $1.831(1.801)$ & \\
\hline & & B3LYP & & $1.553(1.562)$ & $1.872(1.852)$ & \\
\hline & & CAM-B3LYP & & $1.195(1.2457)$ & $1.884(1.879)$ & \\
\hline \multirow[t]{6}{*}{ IBFuEA } & \multirow[t]{3}{*}{ singlet } & HSE & $3.925(3.411)$ & $1.988(1.949)$ & $2.402(2.318)$ & $3.795(3.477)$ \\
\hline & & B3LYP & $3.877(3.138)$ & $2.137(2.087)$ & $2.457(2.376)$ & $4.203(3.600)$ \\
\hline & & CAM-B3LYP & $4.167(3.948)$ & $1.943(1.911)$ & $2.524(2.437)$ & $6.762(6.239)$ \\
\hline & \multirow[t]{3}{*}{ triplet } & HSE & & $0.903(0.951)$ & $1.472(1.458)$ & \\
\hline & & B3LYP & & $1.1542(1.181)$ & $1.555(1.541)$ & \\
\hline & & CAM-B3LYP & & $0.506(0.627)$ & $1.477(1.471)$ & \\
\hline \multirow[t]{6}{*}{ INFuEA } & \multirow[t]{3}{*}{ singlet } & HSE & $3.994(3.393)$ & $2.301(2.291)$ & $2.716(2.668)$ & $3.861(3.445)$ \\
\hline & & B3LYP & $3.938(3.128)$ & $2.431(2.408)$ & $2.745(2.695)$ & $4.263(3.543)$ \\
\hline & & CAM-B3LYP & $4.307(4.139)$ & $2.271(2.269)$ & $2.884(2.843)$ & $6.699(6.132)$ \\
\hline & \multirow[t]{3}{*}{ triplet } & HSE & & $1.475(1.529)$ & $1.905(1.919)$ & \\
\hline & & B3LYP & & $1.641(1.679)$ & $1.957(1.968)$ & \\
\hline & & CAM-B3LYP & & $1.288(1.362)$ & $1.957(1.981)$ & \\
\hline
\end{tabular}


Table S12: Singlet and triplet first excitation energies and homo-lumo gaps for azobenzeneEA (Azo) calculated by time-dependent DFT (TD) and $\triangle$ SCF methods in the singlet and triplet spin multiplicity geometries $(\mathrm{MG}$ ) for various exchange-correlation (xc) functional. Molecular geometries herein are relaxed using the CAM-B3LYP xc-functional. Values outside of parentheses indicate the neutral compound with an ethyl ammonia $\mathrm{NH}_{2}$ tail whereas values in the parentheses indicate the charged species with $\mathrm{NH}_{3}^{+}$. 6-31G* is used for the Gaussian basis set unless otherwise stated. All values are in $\mathrm{eV}$.

\begin{tabular}{l|c|c|c|c|c|c}
\hline & MG & xc-functional & TD $S_{1}$ & TD $T_{1}$ & $\Delta$ SCF $T_{1}$ & Homo-Lumo \\
\hline \multirow{2}{*}{ azoEA } & \multirow{2}{*}{ singlet } & HSE & $2.614(2.516)$ & $1.765(1.698)$ & $1.932(1.869)$ & $3.641(3.491)$ \\
& & B3LYP & $2.598(2.495)$ & $1.825(1.756)$ & $1.977(1.915)$ & $3.990(3.817)$ \\
& & CAM-B3LYP & $2.764(2.692)$ & $1.887(1.835)$ & $2.009(1.956)$ & $6.547(6.426)$ \\
\hline \multirow{6}{*}{ triplet } & HSE & & $-0.253(-0.288)$ & $0.577(0.410)$ & \\
& & B3LYP & & $-0.107(-0.324)$ & $0.494(0.331)$ & \\
& & & $-0.338(-0.339)$ & $0.680(0.494)$ & \\
\hline
\end{tabular}

Table S13: Comparison of $T_{1}$ and $S_{1}$ calculated energy on the B3LYP-relaxed geometries in vacuum and water solvent effects treated by the polarization continuum model. 8

\begin{tabular}{|c|c|c|c|c|c|c|c|}
\hline & & \multicolumn{3}{|l|}{ Vacuum } & \multicolumn{3}{|l|}{ Water } \\
\hline & xc-functional & $S_{1}^{T D}$ & $T_{1}^{T D}$ & $T_{1}^{\triangle S C F}$ & $S_{1}^{T D}$ & $T_{1}^{T D}$ & $T_{1}^{\Delta S C F}$ \\
\hline \multirow[t]{3}{*}{ PEA } & $\mathrm{HSE}$ & 5.498 & 3.54 & 4.338 & 5.506 & 3.777 & 4.353 \\
\hline & B3LYP & 5.429 & 3.755 & 4.433 & 5.436 & 3.534 & 4.447 \\
\hline & CAM-B3LYP & 5.543 & 3.508 & 4.400 & 5.552 & 3.568 & 4.421 \\
\hline \multirow[t]{3}{*}{ AEA } & HSE & 4.457 & 2.563 & 3.106 & 4.437 & 2.582 & 3.114 \\
\hline & B3LYP & 4.396 & 2.728 & 3.136 & 4.377 & 2.743 & 3.143 \\
\hline & CAM-B3LYP & 4.630 & 2.524 & 3.277 & 4.633 & 2.545 & 3.285 \\
\hline \multirow[t]{3}{*}{ AEA } & HSE & 3.333 & 1.685 & 2.136 & 3.304 & 1.697 & 2.139 \\
\hline & B3LYP & 3.293 & 1.841 & 2.172 & 3.265 & 1.850 & 2.174 \\
\hline & CAM-B3LYP & 3.65 & 1.603 & 2.282 & 3.013 & 1.619 & 2.287 \\
\hline \multirow[t]{3}{*}{ PyEA } & HSE & 3.757 & 1.977 & 2.402 & 3.707 & 1.991 & 2.409 \\
\hline & B3LYP & 3.705 & 2.121 & 2.434 & 3.656 & 2.152 & 2.440 \\
\hline & CAM-B3LYP & 3.964 & 1.866 & 2.552 & 3.959 & 1.883 & 2.558 \\
\hline \multirow[t]{3}{*}{ 4MeO-PEA } & HSE & 5.149 & 3.519 & 3.987 & 5.129 & 3.539 & 3.99 \\
\hline & B3LYP & 5.08 & 3.697 & 4.05 & 5.061 & 3.713 & 4.050 \\
\hline & CAM-B3LYP & 5.248 & 3.476 & 4.368 & 5.234 & 3.496 & 4.335 \\
\hline \multirow[t]{3}{*}{$4 \mathrm{NO}_{2}-\mathrm{PEA}$} & HSE & 3.031 & 2.0684 & 2.254 & 2.967 & 2.082 & 2.242 \\
\hline & B3LYP & 2.9653 & 2.136 & 2.287 & 2.878 & 2.136 & 2.265 \\
\hline & CAM-B3LYP & 3.192 & 2.091 & 2.254 & 3.196 & 2.114 & 2.256 \\
\hline \multirow[t]{3}{*}{ 4Br-PEA } & HSE & 5.156 & 3.448 & 4.570 & 5.184 & 3.477 & 4.568 \\
\hline & B3LYP & 5.083 & 3.640 & 4.578 & 5.114 & 3.666 & 4.576 \\
\hline & CAM-B3LYP & 5.363 & 3.4249 & 4.7489 & 5.391 & 3.452 & 4.755 \\
\hline
\end{tabular}




\section{References}

(1) Ishikawa, H.; Noyes, W. A. J Am Chem Soc 1962, 84, 1502-1503.

(2) Ema, K.; Inomata, M.; Kato, Y.; Kunugita, H.; Era, M. Phys Rev Lett 2008, 100, 257401.

(3) Braun, M.; Tuffentsammer, W.; Wachtel, H.; Wolf, H. Chem Phys Lett 1999, 303, 157 $-164$.

(4) Avakian, P.; Merrifield, R. E. Mol Cryst 1968, 5, 37-77.

(5) Braun, M.; Tuffentsammer, W.; Wachtel, H.; Wolf, H. Chem Phys Lett 1999, 307, 373 $-378$.

(6) Joblin, C.; Salama, F.; Allamandola, L. J Chem Phys 1999, 110, 7287-7297.

(7) Flicker, W. M.; Mosher, O. A.; Kuppermann, A. Chem Phys Lett 1976, 38, 489 - 492.

(8) Tomasi, J.; Mennucci, B.; Cammi, R. Chem Revi 2005, 105, 2999-3094. 